\title{
Comparartive Study on Fetal Renal Artery Doppler Waveforms Between Right and Left Sides, At Proximal And Distal Sites Study
}

\author{
Original \\ Article \\ Sherif Dahab, Ahmed Saad, Ahmed Askalany, Hesham Hamza \\ Department of Obstetrics and Gynecology, Cairo University, Kasr Al-Ainy School of Medicine, \\ Cairo University, Egypt
}

\begin{abstract}
Objective: To investigate any variability in fetal renal arteries Doppler indices between right and left sides, proximal and distal site.

Study design Prospective observational study conducted on 150 pregnant women . Renal artery velocity waveforms were measured in both the right and left sides proximally and distally.

Results: The mean PSV was significantly higher at right proximal site $(69.141 \pm 7.6352$ vs. $64.064 \pm 5.0714)$ and at left distal site $(62.261 \pm 4.9222$ vs. $59.841 \pm 4.7978)$, the mean EDV was significantly higher at right proximal $(5.730 \pm$ 1.2253 vs. $5.257 \pm 1.1096$ )and distal sites( $5.27 \pm 1.265$ vs. $4.089 \pm 0.8099)$, The mean PI was significantly higher at right proximal site $(2.3840 .2863 \pm$ vs. $2.170 \pm 0.1953)$ but, distal to the aorta, it was higher on the left side $(2.455 \pm 0.2079$ vs. $2.075 \pm 0.1928)(P$ value $<0.001)$.

There is no correlation between the right and left sides. The mean PSV is significantly higher on the right side

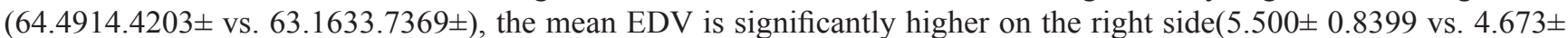
$0.7150)$, the mean RI is higher on the left side $(0.926 \pm 0.0122$ vs. $0.914 \pm 0.0154)$, while the mean PI is also significantly higher on the left side (2.419 \pm 0.1764 vs. $2.122 \pm 0.1324)$.

Conclusion: Renal artery Doppler measurement at a given site cannot be used to predict its opposite.
\end{abstract}

Key Words: doppler indices, fetal renal artery.

Revised: 21 November 2016, Accepted: 1 February 2017

Corresponding Author: Sherif Dahab., Tel.: 01118044888., E-mail: heshamhamza@ebwhs.com

ISSN: 2090-7265, May 2017, Vol. 7, No. 2

\section{INTRODUCTION}

The renal arteries arise directly from the aorta just below the projection of the $12^{\text {th }}$ rib and below the superior mesenteric artery. The left renal artery is usually a little higher and longer than that on the right. The best way to assess the renal arteries during an US scan is to find the abdominal aorta and the renal hilum using a coronal axis view ${ }^{1}$.

The fetal renal artery Doppler waveform proved to have a role in several disorders. Veille \& Kanaan (1989) demonstrated a higher pulsatility index (PI) in growthrestricted fetuses ${ }^{2}$.

Alike, the small for gestational age human fetuses had a higher PI ranges than normal ones ${ }^{3}$. Besides, the peak systolic velocity (PSV) decreased with time in severely growth-restricted foetuses ${ }^{4}$.

The systolic to diastolic ratio (S/D ratio) was significantly higher in past-date pregnancies complicated with oligohydramnios ${ }^{5}$.
Fetal renal artery Doppler pattern proved to be beneficial in detecting many fetal anomalies. Renal agenesis is associated with early and severe oligohydramnios. Most of the time, it is difficult to diagnose it except by absence of fetal renal artery Doppler. A significantly lower PI value was found in Meckel Gruber Syndrome ${ }^{6}$.

In the last two decades, several studies highlighted the importance of the fetal renal artery Doppler in assessing the fetal well-being as well as in monitoring thefetal renalperfusion in different disorders. So, in order to reach a conclusion in abnormal cases, it is crucial to thoroughly study the normal wave pattern of the renal artery. Doppler When assessing Doppler flow of the fetal renal artery, attention must be given to whether flow is best described as plug flow or laminar flow. At the immediate bifurcation of the renal artery with the abdominal aorta, the flow of the blood is essentially constant across the vessel. Theoretically, flow is turbulent at that site and can result in a random, chaotic flow pattern of red blood cells. Closer 
to the renal parenchyma, flow becomes more laminar and assumes a parabolic profile ${ }^{2}$.

In this study, we aimed to investigate any variability in pulsatility/resistance indices and absolute velocities of blood flow in fetal renal arteries dependent on right and left sides, proximal and distal to the aorta..

\section{PATIENTS AND METHODS}

This is a prospective comparative study comparing different Doppler indices of the fetal renal artery on right and left sides at proximal and distal sites between $33+0$ $356+$ weeks of gestation. The study was carried out in Kasr Alainy Hospital in the period between December 2011 and June 2014. The patients were selected randomly from those visiting the Fetal Medicine Unit. A total of 150 cases were enrolled in the study.

The study was approved by local ethics committee. Informed consents about the study, expected value and outcomes and also for Doppler ultrasound examination were obtained.

All participants had singleton pregnancy with no maternal medical disorder diagnosed before or during pregnancy. No identified fetal congenital anomalies excluded by a $2^{\text {nd }}$ trimesteric anomaly scan or anomaly scan at the time of the study. The age of participants ranged from 18 to 35 years and the gestational age ranged from $336+35-0+$ weeks,calculated by a sure last menstrual period or $1^{\text {st }}$ trimesteric US.

We excluded women with medical disorder diagnosed after scan till before delivery and those with poor fetal outcome, as defined by Apgar score at one minute $<7$ or the need of assisted ventilation.

A detailed history was taken; including history of present pregnancy to identify any risk factors associated with this pregnancy as well as recognition of inclusion and exclusion criteria. Besides, thorough physical examination was performed together with US and Doppler study for determination of the gestational age, amniotic fluid index and fetal renal artery Doppler on the right and left sides at two different sites; proximally, close to the aorta, and distally, before any major visible bifurcation of the vessels.

All the recruited women were followed up till delivery to exclude the occurrence of medical disorders or unexplained poor fetal-outcome.The ultrasound and Doppler examination were carried out by the same operator (AS) using a Medison X8 (Medison Co., Ltd. Seoul, Korea) equipped with a 4-7 MHz transabdominal (3D47-EK).

Transabdominal US scan were done with the patient in left lateral position and an axial image of the fetal abdomen was obtained at the level of the fetal kidneys attempting to find an angle of insonation as close as possible to $00^{\circ}$. Intra-uterine growth restriction (IUGR) and macrosomia were excluded by calculating the estimated fetal weight using biparietal diameter, abdominal circumference and femur lengths. Oligo- and polyhydramnios were excluded by measuring the amniotic fluid index (AFI).

Velocity waveforms were sampled in both the right and left renal arteries at two different sites: proximally, close to the aorta, and distally, before any major visible bifurcation of the vessels. Doppler measurements are taken at an angle $<300$. Filter level of $50 \mathrm{MHz}$ was used. A minimum of three consecutive waveforms were used to calculate pulsatility index (PI), resistance index (RI), peak systolic velocity (PSV) and end-diastolic velocity (EDV). These were measured twice at the start as well as at the end of ultrasound examination, and the mean of three measurement was taken.

All subjects were followed up to assure that no denovo conditions appeared and the delivery was uneventful, producing a healthy living neonate. With this foolproof approach, we made sure our values are those of a normal fetus with no influence from a medical condition.

Data were statistically described in terms of mean \pm standard deviation $( \pm$ SD), and range, or frequencies (number of cases) and percentages when appropriate.

Comparison of between the study groups was done using paired $t$ test. Agreement across sides of measurements within the individuals was analyzed as $95 \%$ limits of agreement, i.e. the mean of the difference between two locations $\pm 2 \mathrm{SD}$. The consistency in the ranking of the subjects across sites and sides of measurements was analyzed using Spearman's correlation coefficient. The intraobserver variation was analyzed as the intraclass correlation coefficient (ICC) by means of randomeffects regression. $\mathrm{p}$ values less than 0.05 was considered statistically significant.

All statistical calculations were done using computer programs SPSS (Statistical Package for the Social Science; SPSS Inc., Chicago, IL, USA) version 15 for Microsoft Windows.

\section{RESULTS}

Demographic data of the studied population including age, parity and gestational age are presented in Table 1.

The statistical values of biparietal diameter, abdominal circumference, femur length, estimated fetal weight (Hadlock's formula) and amniotic fluid index are presented in table 1 . All were between $5^{\text {th }}$ and $95^{\text {th }}$ percentile of the corresponding gestational age. 
Table 2 shows the mean and SD of Doppler indices comparing each individual site with its correspondent.

Apart from the RI at the proximal site between right and left sides, whose, $p$ value was 0.640 , all comparisons were statistically different ( $p$ value $<0.05$ ).

The mean PSV was significantly higher at right proximal site and at left distal site. Alike, the mean EDV was significantly higher at right proximal and distal sites. Besides, the mean RI was almost the same at the proximal sites but higher on the left distal site, while the mean PI was significantly higher at right proximal site but, distal to the aorta, it was higher on the left side (Table 2).

Using intraclass correlation coefficient (ICC), we found that, when comparing corresponding sites, all the indices had very poor correlation. While some results had positive correlations, others negative correlations, and all values were near zero (Table 2 ).

Figures 1- 4 show diagrammatic illustrations of the ICC and give an idea on how values were scattered from each other. The $p$ values were way above 0.05 , which ensures no correlation between the measurements. In all the graphs, the $\mathrm{x}-$ axis represents the right side while the $\mathrm{y}-$ axis represents the left side Table 3 compared the same Doppler indices but this time between all of the measurements on the right side (proximal and distal) with all of the measurements on the left side (proximal and distal). In such comparison we aimed to see if there is a trend in any

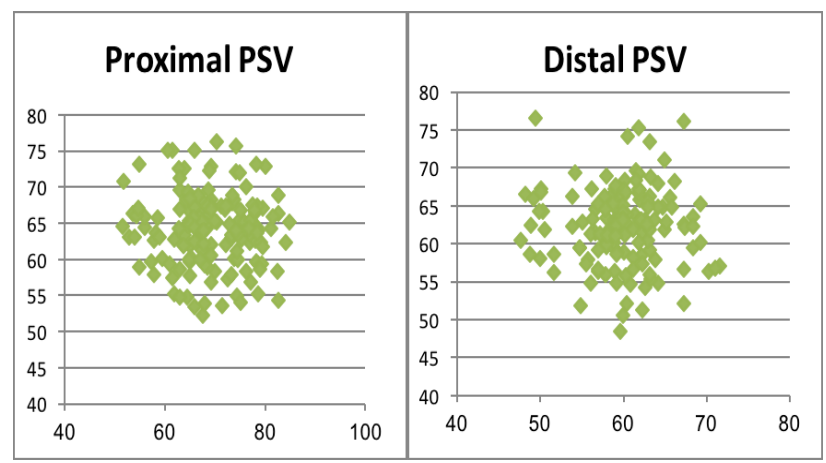

Fig. 1: Proximal and distal PSV values at right and left sides (ICC -0.060 and -0.033 ).

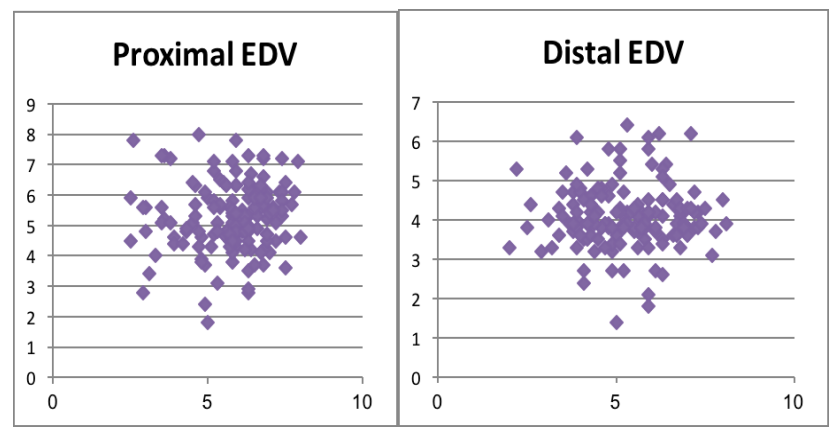

Fig. 2: Proximal and distal EDV values at right and left sides (ICC 0.068 and 0.010 ). of the four indices when measured at the right and left fetal renal arteries. Again, there was no correlation between the two sides and all ICC were near zero.

The mean PSV was significantly higher on the right side, the mean EDV was also significantly higher on the right side, the mean RI was higher on the left side when compared to the right side. Also, the mean PI was significantly higher on the left side compared to the right side (Table 3). We compared the four Doppler indices between proximal sites (right and left together) and distal sites (right and left together). The aim of such a comparison was to detect a trend in the indices when the FRA Doppler is measured proximal and distal to the aorta, regardless of the side. Again, no correlation was determined between the measurements and the ICC was near zero (Table 4, Figure 5).

The PSV significantly decreased "as it goes from proximal to distal", the mean EDV also decreased significantly "as it goes from proximal to distal", the mean RI significantly rose as it goes from proximal to distal while the mean PI remained almost unchanged, with a very small insignificant drop as blood passes from proximal to distal (Table 4).

The intraobserver variations measured as ICC $(\mathrm{CV}, 95 \%$ limits of agreement) were $0.79(-11.98$ to $14.66 \mathrm{~cm} / \mathrm{s})$ for PSV, $0.79(-4.1$ to $4.1 \mathrm{~cm} / \mathrm{s})$ for EDV, 0.85 ( -0.05 to 0.05$)$ for RI and 0.67 ( -0.41 to 0.46$)$ for PI.
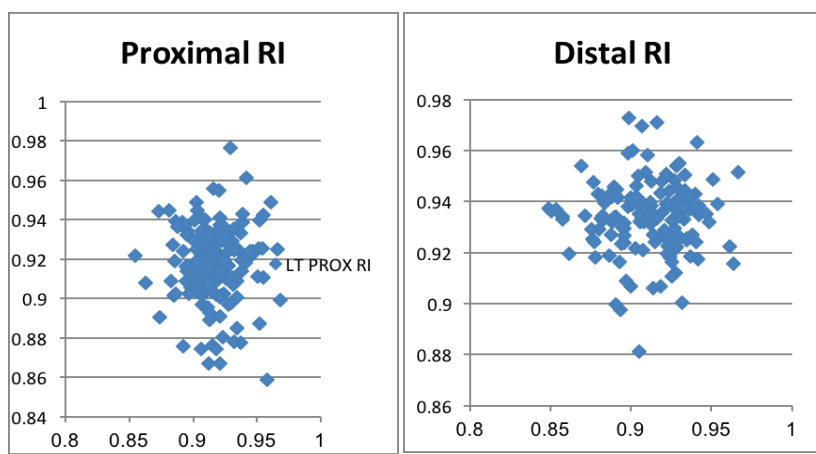

Fig. 3: Proximal and distal RI values at right and left sides (ICC 0.016 and 0.012 )

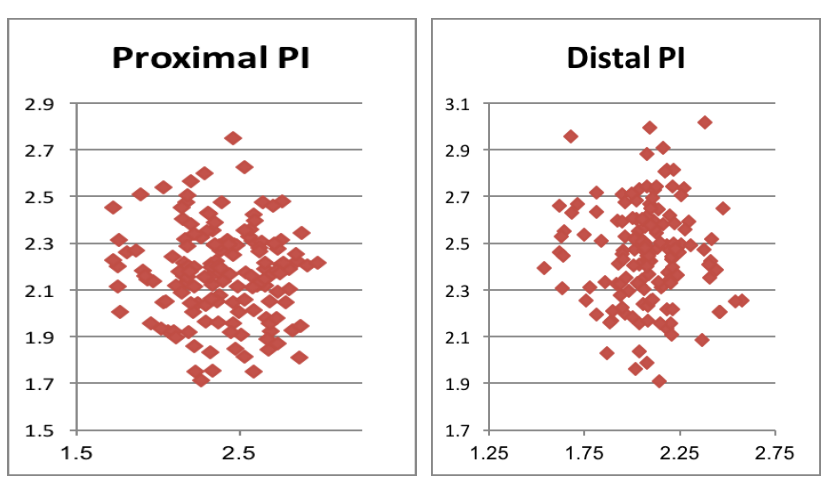

Fig. 4: Proximal and distal PI values at right and left sides (ICC -0.073 and -0.034$)$ 

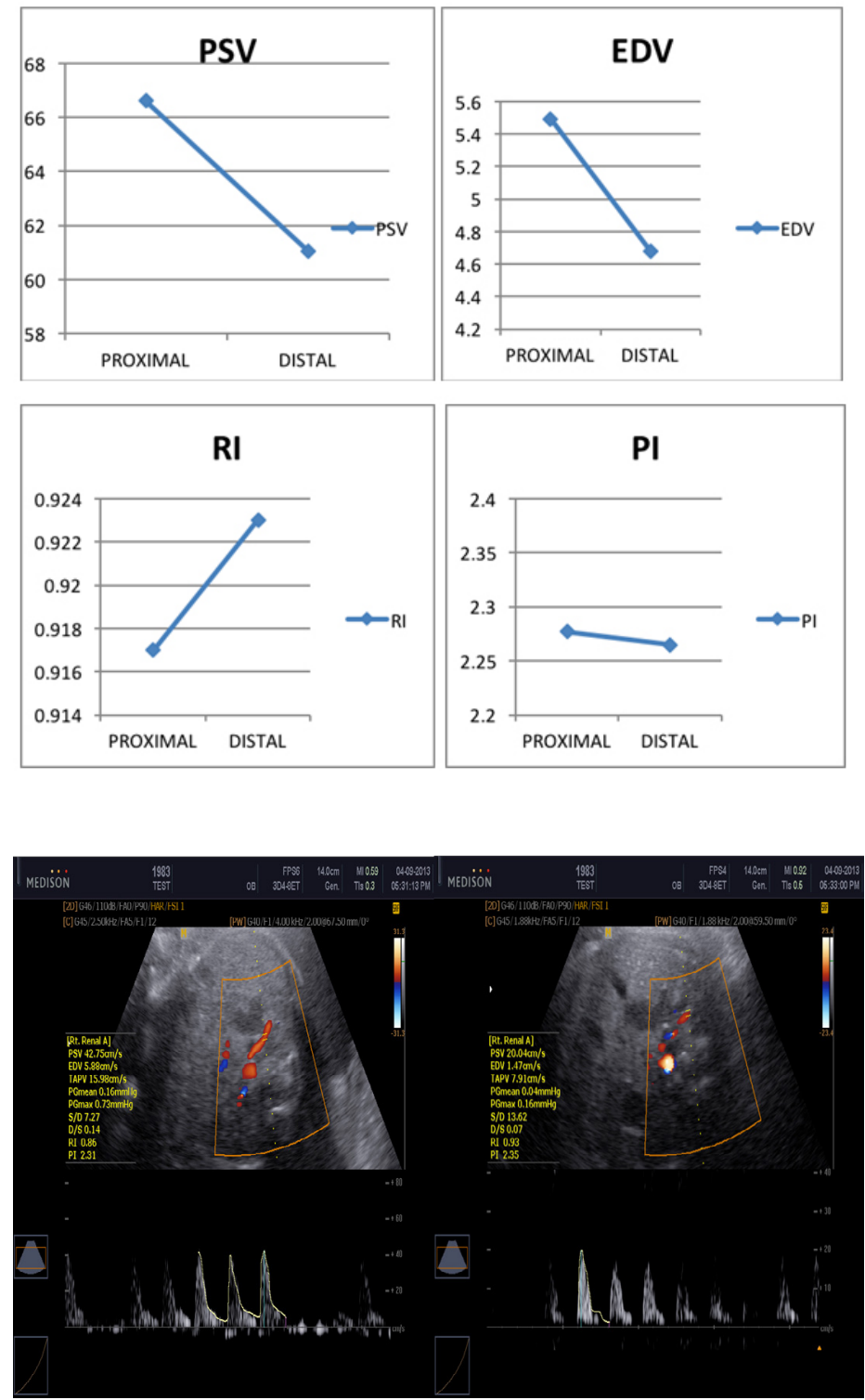

Fig. 5: Mean PSV, EDV, RI, PI at proximal and distal sides (Rt and Lt together).
Fig. 6: Doppler measurements at right proximal (A) and right distal (B) sites. the measurements show a higher absolute velocities at proximal sites and higher RI at distal sites.

Table 1: Characteristic data of the studied population.

\begin{tabular}{lccc}
\hline & & Mean \pm SD & Range \\
\hline Age (years) & & $2.6 \pm 4.79$ & $18-35$ \\
Parity & & $1.3 \pm 2.4$ & $0-5$ \\
GA (days) & & $240.9 \pm 5.67$ & $232-251$ \\
& BPD (mm) & $84.91 \pm 2.286$ & $79-91$ \\
Ultrasonographic & AC (mm) & $293.56 \pm 13.408$ & $265-320$ \\
fetal parameters & FL $(\mathrm{mm})$ & $64.76 \pm 1.963$ & $60-69$ \\
& EFW (gm) & $2,302.68 \pm 279.598$ & $1767-2980$ \\
& AFI $(\mathrm{cm})$ & $12.80 \pm 2.326$ & $7-19$ \\
\hline
\end{tabular}

GA Gestational age

BPD Biparietal diameter

AC abdominal circumference

FL femur length

EFW Estimated fetal weight

AFI Amniotic fluid index 
Dahab et al, .

Table 2: Statistical analysis if Doppler indices at different sites.

\begin{tabular}{|c|c|c|c|c|c|c|}
\hline & Mean \pm SD & Range & $P$ value & $95 \% \mathrm{CI}$ & $\mathrm{ICC}$ & $P$ value \\
\hline RT PROX PSV & $69.141 \pm 7.6352$ & $51.6-84.9$ & \multirow{2}{*}{0.000} & \multirow{2}{*}{$\begin{array}{r}(3.5575)- \\
(6.5958)\end{array}$} & \multirow{2}{*}{-0.060} & \multirow{2}{*}{0.466} \\
\hline LT PROX PSV & $64.064 \pm 5.0714$ & $52.3-76.3$ & & & & \\
\hline RT DIST PSV & $59.841 \pm 4.7978$ & $47.6-71.6$ & \multirow{2}{*}{0.000} & \multirow{2}{*}{$(-3.5474)-(-1.2926)$} & \multirow{2}{*}{-0.033} & \multirow{2}{*}{0.685} \\
\hline LT DIST PSV & $62.261 \pm 4.9222$ & $48.5-76.5$ & & & & \\
\hline RT PROX EDV & $5.730 \pm 1.2253$ & $2.5-8$ & \multirow{2}{*}{0.000} & \multirow{2}{*}{$(0.2159)-(0.7308)$} & \multirow{2}{*}{0.068} & \multirow{2}{*}{0.405} \\
\hline LT PROX EDV & $5.257 \pm 1.1096$ & $1.8-8$ & & & & \\
\hline RT DIST EDV & $5.27 \pm 1.265$ & $2-8$ & \multirow{2}{*}{0.000} & \multirow{2}{*}{$(0.9407)-(1.4233)$} & \multirow{2}{*}{0.010} & \multirow{2}{*}{0.904} \\
\hline LT DIST EDV & $4.089 \pm 0.8099$ & $1.4-6.4$ & & & & \\
\hline RT PROX RI & $0.916 \pm 0.0201$ & $0.9-1$ & \multirow{2}{*}{0.640} & \multirow{2}{*}{$(-0.0056)-(0.0035)$} & \multirow{2}{*}{-0.016} & \multirow{2}{*}{0.846} \\
\hline LT PROX RI & $0.917 \pm 0.0194$ & $0.9-1$ & & & & \\
\hline RT DIST RI & $0.911 \pm 0.0235$ & $0.8-1$ & \multirow{2}{*}{0.000} & \multirow{2}{*}{$\begin{array}{c}(-0.0272)- \\
(-0.0184)\end{array}$} & \multirow{2}{*}{0.012} & \multirow{2}{*}{0.886} \\
\hline LT DIST RI & $0.934 \pm 0.0138$ & $0.9-1$ & & & & \\
\hline RT PROX PI & $2.384 \pm 0.2863$ & $1.7-3$ & \multirow{2}{*}{0.000} & \multirow{2}{*}{$(0.1561)-(0.2716)$} & \multirow{2}{*}{-0.073} & \multirow{2}{*}{0.377} \\
\hline LT PROX PI & $2.170 \pm 0.1953$ & $1.7-2.7$ & & & & \\
\hline RT DIST PI & $2.075 \pm 0.1928$ & $1.5-2.6$ & \multirow{2}{*}{0.000} & \multirow{2}{*}{$(-0.4261)-(-0.3331)$} & \multirow{2}{*}{-0.034} & \multirow{2}{*}{0.678} \\
\hline LT DIST PI & $2.455 \pm 0.2079$ & $1.9-3$ & & & & \\
\hline
\end{tabular}

Rt right

Lt left

Prox proximal

Dist distal

PSV Peak systolic volume EDV End diastolic volume RI resistance index

PI Pulsatility index

Table 3: Comparison of indices of right and left sides (proximal and distal together).

\begin{tabular}{|c|c|c|c|c|c|c|c|}
\hline & Mean $\pm \mathrm{SD}$ & Range & $P$ value & Mean difference & $95 \% \mathrm{CI}$ & ICC & $P$ value \\
\hline RT PSV & $64.491 \pm 4.4203$ & $47.6-84.9$ & \multirow{2}{*}{0.008} & \multirow{2}{*}{1.3283} & \multirow{2}{*}{$(0.3577)-(2.2990)$} & \multirow[b]{2}{*}{-0.081} & \multirow[b]{2}{*}{0.322} \\
\hline LT PSV & $63.163 \pm 3.7369$ & $48.5-76.5$ & & & & & \\
\hline RT EDV & $5.500 \pm 0.8399$ & $2-8$ & \multirow{2}{*}{0.000} & \multirow{2}{*}{0.8277} & \multirow{2}{*}{$(0.6423)-(1.0131)$} & \multirow[b]{2}{*}{-0.086} & \multirow[b]{2}{*}{0.293} \\
\hline LT EDV & $4.673 \pm 0.7150$ & $1.4-8$ & & & & & \\
\hline RT RI & $0.914 \pm 0.0154$ & $0.8-1$ & \multirow{2}{*}{0.000} & \multirow{2}{*}{-0.0119} & \multirow{2}{*}{$(-0.0153)-(-0.0086)$} & \multirow[b]{2}{*}{-0.114} & \multirow[b]{2}{*}{0.163} \\
\hline LT RI & $0.926 \pm 0.0122$ & $0.9-1$ & & & & & \\
\hline RT PI & $2.122 \pm 0.1324$ & $1.5-2.6$ & \multirow{2}{*}{0.000} & \multirow{2}{*}{0.2967} & \multirow{2}{*}{$(0.2608)-(0.3327)$} & \multirow[b]{2}{*}{-0.021} & \multirow[b]{2}{*}{0.797} \\
\hline LT PI & $2.419 \pm 0.1764$ & $1.7-2.7$ & & & & & \\
\hline
\end{tabular}

Table 4: Doppler indices at proximal and distal sites (right and left together).

\begin{tabular}{|c|c|c|c|c|c|c|c|}
\hline & Mean \pm SD & Range & $P$ value & $\begin{array}{c}\text { Mean differen } \\
\text { ce }\end{array}$ & $95 \% \mathrm{CI}$ & ICC & $P$ value \\
\hline Prox PSV & $66.602 \pm 4.4545$ & $51.6-84.9$ & \multirow{2}{*}{0.000} & \multirow{2}{*}{$\begin{array}{c}5.5510 \\
0\end{array}$} & \multirow{2}{*}{$(4.64250)-(6.45950)$} & \multirow[b]{2}{*}{-0.015} & \multirow[b]{2}{*}{0.857} \\
\hline Distal PSV & $61.051 \pm 3.3790$ & $47.6-76.5$ & & & & & \\
\hline Prox EDV & $5.493 \pm 0.8542$ & $1.8-8$ & \multirow{2}{*}{0.000} & \multirow{2}{*}{0.8137} & \multirow{2}{*}{$(0.6171)-(1.0103)$} & \multirow[b]{2}{*}{-0.144} & \multirow[b]{2}{*}{0.078} \\
\hline Distal EDV & $4.680 \pm 0.7545$ & $1.4-8$ & & & & & \\
\hline Prox RI & $0.917 \pm 0.0139$ & $0.9-1$ & \multirow{2}{*}{0.001} & \multirow{2}{*}{-0.0058} & \multirow{2}{*}{$(-0.0091)-(-0.0025)$} & \multirow[b]{2}{*}{-0.101} & \multirow[b]{2}{*}{0.219} \\
\hline Distal RI & $0.923 \pm 0.0137$ & $0.8-1$ & & & & & \\
\hline Prox PI & $2.277 \pm 0.1673$ & $1.7-2.6$ & \multirow{2}{*}{0.492} & \multirow{2}{*}{0.0122} & \multirow{2}{*}{$(-0.0228)-(0.0472)$} & \multirow[b]{2}{*}{0.005} & \multirow[b]{2}{*}{0.948} \\
\hline Distal PI & $2.265 \pm 0.1393$ & $1.5-2.7$ & & & & & \\
\hline
\end{tabular}




\section{DISCUSSION}

In our study, we obtained significant differences in the Doppler indices at different sites over the renal artery. In general, the velocity of blood is faster near its origin, while the resistance increases near the kidneys. The PI is the least to show changes at different sites. Subsequently, it is the recommended index to consider while evaluating the fetal renal blood flow. We recommend using the right side (bearing in mind that PSV and EDV are slightly higher) either proximal or distal.

When comparing proximal to distal, the PI did not change significantly. When comparing right and left sides, the PI was higher on the left side. Most of the disorders had a higher than normal PI value. So it seems reasonable to avoid the already high side (left) and to measure at the low side (right). When in doubt, readings on both sides should be considered.

We made our observation on 150 cases with the required inclusion criteria. While, previous trials included lower numbers, as Veille et al. (1993) studied only 22 cases $^{5}$ and Vyas et al. (1989) enrolled 114 cases $^{7}$.

But, in their work, the sample range was from 17 to 43 weeks and their measurements taken from a single site and aimed at detecting changes in FRA Doppler throughout pregnancy. Also, a high-pass filter of $125 \mathrm{~Hz}$ was used which may have obstructed low levels of enddiastolic flow. Akita et al. (1991) recruited 102 cases over a wide range of gestations - 20 - 40 weeks7. Haugen et al. (2004) carried out a similar research on 27 fetuses only "almost 15/ our sample size".

Regarding the sample, our current research investigated 150 cases, which is comparatively a large sample. So, despite being different, our results, could have a heavier impact than theirs ${ }^{8}$.

Noteworthy, we chose $33+0$ to $35+6$ weeks of gestation for several reasons. First, most obstetricians consider such gestational ages the true critical time in deciding whether or not to terminated pregnancy in fetal and maternal morbidities. Although fetal viability in Egypt is around 28 weeks, the best chance of neonatal survival is around 34 weeks. Second, corticosteroids are best effective up to $34+6$ weeks 9 . Third, the perinatal mortality and/or morbidity are much higher before 34 weeks than after 34 weeks ${ }^{10}$.

The aforementioned justifies our selection of this gestational age for our study.

When comparing the Doppler indices near and distal to the aorta, PSV and EDV were higher at proximal sites and lower at distal sites. PSV and EDV represent the speed of blood flow. Since, blood flow is faster near the aorta than near the kidneys. The RI increased significantly further along the FRA. Close to the renal hilum the renal arteries divide into multiple branches with large anterior and posterior branches. These branches in turn divide into large segment arteries, which eventually terminate in arcuate arteries ${ }^{11}$. So, that further along the fetal renal artery, the vessel decreases in diameter which is reflected as an increase in the vascular resistance. The PI, however, remained relatively constant. We, therefore, recommend PI as the most reliable index in measuring the FRA Doppler.

Consistent with the latest International Society of Ultrasound in Obstetrics and Gynecology (ISUOG) guidelines, which recommends the pulsatility index as the commonly used index in current practice ${ }^{12}$.

When comparing right and left FRA, we found the PSV and EDV significantly higher on the right side. Interestingly, the RI was significantly higher on the left side, but the PI was higher on the right side, indicating that while blood flow is faster on the right side but gives a biased idea about the vascular resistance difference between both sides. The left renal artery is usually a little higher and longer than that on the right ${ }^{11}$. Therefore, further investigations in terms of fetal anatomy are necessary to correlate the aforementioned with our results.

To our knowledge, there is no reference of a detailed description of the anatomy of the fetal renal artery and so, the validity of our results depends on the comparison with others' studies. Similar to our results, Haugen et al. found that the PSV and EDV are higher at the proximal sites, and that the EDV is higher on the right side. They found no difference in the PSV, RI and PI at right and left sides. We do not question the validity of Haugen et al. results, yet we question their sample size. Statistically speaking, the larger the sample size, the less the error of chance. A sample of 27 fetuses brings into a question of how strong such data is. On the other hand, we included 150 fetuses, which left little room for error ${ }^{9}$

Vyas et al. (1989) did not specify the site of sampling ${ }^{7}$, while Veille and Kanaan chose to sample distal to the aorta ${ }^{2}$.

In our study, all ICC values were near zero, regardless positive or negative.

This means, none of the Dop+pler indices have a linear correlation with its correspondent. In other words, a Doppler measurement at a given site cannot be used to predict the Doppler value at its opposite site. Haugen used the interclass correlation to describe his data. Since more than one operator took the measurements, it was essential to calculate the interclass correlation to reflect low measurement error 
between different operators. In our study, we used the intraclass correlation since all measurements were taken by the same operator.

The fetal renal artery is a very important artery. It has a proven role in kidney-related disorders and also in measuring the amount of liquor around the fetus.

However, it is not validated to use as a strong predictor of the fetal wellbeing. Yet, the idea of measuring the fetal renal artery Doppler to assess the fetal condition and to make the possible plans of management in high risk pregnancies, is not far from reality. The fact that there are two renal arteries raises the question - where should the measurement be taken?

Normal variations in the fetal renal artery Doppler needs to be thoroughly evaluated with larger number of cases at different gestational ages, studying their correlations with the amniotic fluid volume, fetal-gender, and renal volume.

\section{CONFLICT OF INTERESTS}

There are no conflicts of interest.

\section{REFERENCES}

1. Veille JC. Pulsed Doppler ultrasonography of the human fetal renal artery In Doppler ultrasound in obstetrics and gynecology 2nd ed. Maulik D (ed). Springer- Verlag Berlin Heidelberg. 2005. Ch 15; PP.211- 226.

2. Veille, J. C., Kanaan C. Duplex Doppler ultrasonographic evaluation of the fetal renal artery in normal and abnormal fetuses. Am J Obst gynecol. 1989 ;161(6 Pt 1): 1502-1507.

3. Campbell, S., S. Vyas, and K. H. Nicolaides. Doppler investigation of the fetal circulation. J Perinat Med 1991; 19 (1 -2): 21-26.

4. Stigter RH, Mulder EJ, Bruinse HW, Visser GH. Doppler studies on the fetal renal artery in the severely growth-restricted fetus. Ultrasound Obstet Gynecol 2001: 18(2): 141-145.
5. Veille JC, Hanson RA, Tatum K, Kelley K Quantitative assessment of human fetal renal blood flow. Am J Obst Gynecol.1993: 169(6):1399-1402.

6. Azpurua H, Dulay AT, Buhimschi IA, Bahtiyar MO, Funai E, Abdel- Razeq SS, et al. Fetal renal artery impedance as assessed by Doppler ultrasound in pregnancies complicated by intraamniotic inflammation and preterm birth. Am J Obst gynecol 2009: 200(2):203.e1-203.e11.

7. Vyas S., Nicolaides KH, Campbell S. Renal artery flowvelocity waveforms in normal and hypoxemic fetuses. Am J Obst gynecol 1989: 161(1):168-172.

8. Akita, A., Okada O, Saito T, Koresawa M, Kato H. [Evaluation of the renal artery in the fetuses with growth retardation and oligohydramnios by two dimensional Doppler ultrasonography]. Nihon Sanka Fujinka Gakkai zasshi 1991: 43(11): 1554-1560.

9. Haugen, G., Godfrey K, Crozier S, Hanson M. Doppler blood flow velocity waveforms in the fetal renal arteries: Variability at proximal and distal sites in the right and left arteries. Ultrasound Obstet Gynecol 2004: 23(6):590-593.

10. Roberts D, Dalziel S. Antenatal corticosteroids for accelerating fetal lung maturation for women at risk of preterm birth. The Cochrane database of systematic reviews 2006 ; (3): Article ID CD004454.

11. Lisonkova S, Sabr Y, Butler B, Joseph KS. International comparisons of preterm birth: Higher rates of late preterm birth are associated with lower rates of stillbirth and neonatal death. BJOG 2012: 119(13):1630-1639.

12. International Society of Ultrasound in Obstetrics and Gynecology, Carvalho JS, Allan LD, Chaoui R, Copel JA, DeVore GR, et al. ISUOG Practice Guidelines (updated): Sonographic screening examination of the fetal heart. Ultrasound Obstet Gynecol 2013: 41(3):348-359. 